05

\title{
Влияние скин-эффекта и активных потерь на интенсивность линий ЭПР в полупроводящих веществах
}

\author{
() А.М. Зюзин, А.А. Карпеев, Н.В. Янцен \\ Мордовский государственный университет им. Н.П. Огарева, \\ Саранск, Россия \\ E-mail: zyuzin.am@rambler.ru
}

Поступила в Редакцию 24 сентября 2019 г.

В окончательной редакции 4 октября 2019 г.

Принята к публикации 8 октября 2019 г.

Исследовано влияние скин-эффекта и активных потерь в образцах полупроводящего композита с широким диапазоном значений проводимости на интенсивность линии поглощения ЭПР. Предложен подход, позволяющий получить удовлетворительное соответствие расчетных и экспериментальных результатов. Показано, что в зависимости от проводимости исследуемого вещества, интенсивность линии поглощения, соответствующая единице объема, может в разы уменьшаться с увеличением объема исследуемого образца.

Ключевые слова: электронный парамагнитный резонанс, скин-эффект, полупроводящие вещества.

DOI: 10.21883/FTT.2020.02.48875.590

\section{1. Введение}

Важное место в ряду веществ, исследуемых методом ЭПР, занимают материалы, обладающие заметной электрической проводимостью. К числу последних относятся, в частности, углеродосодержащие полимерные композиты $[1,2]$, которые находят все более широкое практическое применение в различных областях, например при изготовлении экранных оболочек высоковольтных кабелей, саморегулирующихся нагревательных кабелей, электромагнитных экранирующих и поглощающих покрытий и ряде других. В качестве наполнителей, используемых для обеспечения электропроводящих свойств таких композитов чаще всего используется технический углерод и углеродные нанотрубки [3-5]. Присутствие последних в составе полимерного композита обуславливает не только электропроводность, но и достаточно интенсивный сигнал ЭПР, что свидетельствует о наличии неспаренных электронов в структуре частиц технического углерода и углеродных нанотрубок [6-9]. Это может быть использовано для исследования электронного состояния, механизмов проводимости и, например, определения концентраций технического углерода или углеродных нанотрубок в составе композита. Для определения концентрации $N$ парамагнитных центров используется методика, основанная на сопоставлении площади под кривой поглощения (интегральной интенсивности) исследуемого и эталонного образцов. В качестве последнего обычно выбирают диэлектрическое парамагнитное вещество. Если линии поглощения ЭПР аппроксимируются одной формой, например лоренцевой или гауссовой, и спектры регистрируются при одинаковых условиях, то концентрацию парамагнитных центров можно определить с помощью соотношения [10]:

$$
N=N_{0} \frac{V_{0} I^{\prime} \Delta H_{p p}^{2}}{V_{0} I_{0}^{\prime} \Delta H_{p p 0}^{2}},
$$

где $N_{0}-$ концентрация парамагнитных центров для эталонного образца, $V$ - объем образца, $I^{\prime}-$ амплитуда производной линии поглощения, $\Delta H_{p p}-$ ширина линии (расстояние по шкале полей между пиками этой производной).

По интенсивности и ширине линий ферромагнитного резонанса можно определять и намагниченность насыщения ферромагнетика, в том числе и анизотропного $[11,12]$.

На интенсивность линии поглощения в полупроводящих веществах, кроме резонансного значения мнимой части высокочастотной восприимчивости и объема образца, большое влияние оказывают следующие факторы: скин-эффект, активные и диэлектрические потери, которые приводят к дополнительному вкладу в добротность системы резонатор-образец, а также потери, связанными с резонансным поглощением. Для обеспечения необходимой точности измерений, а также корректной интерпретации зависимостей концентрации парамагнитных центров в веществах, в которых имеет место достаточно сильная зависимость удельного сопротивления, например от температуры или иных параметров, весьма важным является учет влияния вышеперечисленных факторов на интенсивность линии поглощения. Влияние скин-эффекта в металлических образцах на спектры ЭПР изучено достаточно подробно $[13,14]$, однако в полупроводящих веществах со специфическими механизмами проводимости, к которым относятся и углеродсодержащие композиты, влияние скин-эффекта на характеристики ЭПР остается малоизученным. 


\section{2. Влияние скин-эфффекта на интенсивность линии ЭПР}

Влияние скин-эффекта начинает существенно проявляться когда вещество обладает достаточно заметной электропроводностью (удельное сопротивление $\left.\rho \lesssim 10^{4} \Omega \cdot \mathrm{cm}\right)$. В этом случае микроволновое поле $h$ не будет однородным по всему объему образца, а будет уменьшаться внутрь от его поверхности. Это эквивалентно тому, что объем образца эффективно взаимодействующий с полем $h$ уменьшается. Такое уменьшение можно рассматривать как уменьшение коэффициента заполнения образцом объема резонатора спектрометра $\eta$, значению которого пропорциональна амплитуда сигнала ЭПР $[10,15]$.

$$
I \propto \eta \chi_{r}^{\prime \prime} Q_{u}
$$

где $Q_{u}$ - добротность системы резонатор-образец.

Таким образом влияние скин-эффекта ведет к уменьшению интенсивности линии поглощения ЭПР $I$, амплитуды ее производной $I^{\prime}$ и интегральной интенсивности (площади под кривой поглощения), которые прямо связаны между собой. По этой причине величина интенсивности, соответствующая единице объема образца (удельная интенсивность) $J=I / V$, будет зависеть от объема этого образца и глубины скин-слоя $\Delta$

$$
\Delta=\sqrt{2 \rho / \omega \mu \mu_{0}}
$$

где $\rho-$ удельное объемное сопротивление, $\omega-$ круговая частота, $\mu-$ магнитная проницаемость, $\mu_{0}-$ магнитная постоянная. С увеличением $V \propto l^{3}(l-$ линейные размеры образца) влияние скин-эффекта на удельную интенсивность линии поглощения ЭПР будет сказываться во все большой степени.

Проявление скин-эффекта при воздействии стоячей электромагнитной волны на образец, находящийся в резонаторе радиоспектрометра и его влияние на величину сигнала ЭПР будет на наш взгляд обладать определенной спецификой по сравнению, например, с классическим случаем возрастания сопротивления проводника с переменным током, когда плотность тока уменьшается с расстоянием $x$ от его поверхности как $j=j_{0} \exp (-x / \Delta)$. Таким же образом изменяется и напряженность $E$ электрического поля внутрь образца, поскольку $\mathbf{j}=\mathbf{E} / \rho$.

Будем рассматривать стоячую электромагнитную волну в резонаторе как суперпозицию падающей и отраженной волн. Пусть поле $h(x)$ волны, распространяющейся внутрь образца, из-за действия скин-эффекта затухает с расстоянием $x$ от поверхности как $h(x)=h_{0} \exp (-x / \Delta)$. Поле сигнала, отраженного от элемента объема $d V$, находящегося на глубине $x$, а следовательно и величина его изменения, обусловленного резонансным поглощением, в результате обратного прохождения к поверхности образца будет также затухать как $\exp (-x / \Delta)$. Таким образом, результирующее ослабление величины изменения поля $h_{0}$, обусловленное резонансным поглощением системой парамагнитных центров в объеме образца $d V$ на глубине $x$ от его поверхности будет определяться множителем $\exp (-2 x / \Delta)$. Это эквивалентно тому, что путь, на котором происходит затухание, удваивается.

Плотность мощности микроволнового поля, поглощаемой в образце при резонансе можно выразить как $[10,15,16]$ :

$$
w=\frac{\omega}{2} \chi^{\prime \prime} \cdot h^{2}
$$

где $\omega-$ круговая частота микроволнового поля.

В результате интенсивность сигнала ЭПР можно представить, как

$$
I \propto \frac{\omega}{2} \chi_{r}^{\prime \prime} \cdot h_{0}^{2} \cdot \int_{\text {surf }}^{0} \exp (-4 x / \Delta) d V=\frac{\omega}{2} \chi_{r}^{\prime \prime} \cdot h_{0}^{2} \cdot V \cdot \beta(V),
$$

где $\beta(V)=\frac{1}{V} \int_{\text {surf }}^{0} \exp (-4 x / \Delta) d V$ - коэффициент учитывающий влияние скинэффекта на удельную интенсивность линии поглощения ЭПР и зависящий от объема образца, точка $O$ соответствует геометрическому центру образца.

Нами был проведен расчет коэффициента $\beta(V)$ для часто используемых форм образцов - шарообразной и кубической. Подобный расчет легко может быть сделан и для образцов (в том числе и жидких) имеющих, например, форму параллелепипеда (пластинка или жидкость в кювете), цилиндра (капилляр) или других.

Для образцов шарообразной формы расчет коэффициента $\beta(V)$, производили с помощью выражения

$$
\beta(V)=\frac{3}{4 \pi R^{3}} \int_{0}^{R} 4 \pi r^{2} \exp \left[\frac{-4(R-r)}{\Delta}\right] d r
$$

где $R$ - радиус шара.

Для образцов кубической формы

$$
\beta(V)=\frac{24}{a^{3}} \int_{0}^{a / 2} x^{2} \exp \left[\frac{-4\left(\frac{a}{2}-x\right)}{\Delta}\right] d x,
$$

где $a-$ длина ребра куба. Понятно, что при одинаковом объеме $V$, значения $\beta$ будут несколько большими для образцов кубической формы по сравнению с шарообразными.

Поскольку в экспериментах и при расчете $N$ или намагниченности иногда более удобно оперировать не объемом, а массой образца, то выражения (6) и (7) могут быть легко преобразованы в зависимости $\beta(m)$.

\section{3. Влияние активных потерь на интенсивность линии ЭПР}

Другой важный фактор, влияющий на интенсивность линии ЭПР, связан с зависимостью от объема образца 
величины вклада в добротность $Q_{u}$ системы резонаторобразец, обусловленного диэлектрическими и активными потерями в образце, а также резонансным поглощением. Это следует из выражения (2) в соответствии с которым интенсивность сигнала поглощения пропорциональна добротности системы резонатор-образец $Q_{u}$. Последняя связана с добротностью ненагруженного резонатора с держателем образца $Q_{0}$, и вкладом в величину добротности, обусловленным диэлектрическими и активными потерями в образце $Q_{s}$, как

$$
\frac{1}{Q_{u}}=\frac{1}{Q_{0}}+\frac{1}{Q_{s}} .
$$

Как следует из результатов эксперимента, диэлектрические потери матрицы исследуемого композита (этиленвинилацетата) на частоте $9.3 \mathrm{GHz}$ являются весьма малыми и, поэтому, в расчетах ими пренебрегали. Также, ввиду малости, не учитывался вклад в добротность, обусловленный резонансным поглощением. Отметим, что влияние этого вклада обратно пропорционально ширине линии поглощения, которая составляла $38 \mathrm{Oe}$.

Вклад в величину добротности, обусловленный активными потерями в образце, можно в первом приближении представить, как

$$
Q_{s}=\frac{K}{V}
$$

где $K$ - некоторый параметр, зависящий в основном от проводимости образца, $V$ - объем образца. Понятно, что величина активных потерь будет зависеть и от скинэффекта. Из (8) и (9) следует, что коэффициент, учитывающий влияние изменения добротности $Q_{u}$ из-за действия активных потерь в образце на амплитуду сигнала поглощения, будет равен

$$
\lambda(V)=\frac{Q_{u}}{Q_{0}}=\frac{K}{K+Q_{0} V} .
$$

В расчетах значение $Q_{0}$ принималось равным 3000 .

Таким образом, из вышеизложенного следует, что регистрируемая интенсивность линии поглощения ЭПР полупроводящего вещества будет пропорциональна следующему выражению

$$
I \propto \frac{\omega}{2} \chi_{r}^{\prime \prime} \cdot h_{0}^{2} \cdot V \cdot \beta(V) \cdot \lambda(V),
$$

а удельная соответственно

$$
I \propto \frac{\omega}{2} \chi_{r}^{\prime \prime} \cdot h_{0}^{2} \cdot \beta(V) \cdot \lambda(V)
$$

\section{4. Эксперимент}

Эксперимент проводился на образцах полупроводящего композита матрицей которого являлся этиленвинилацетат, а наполнителем обеспечивающим электропроводность технический углерод. Объемная плотность образцов зависела от содержания технического углерода и была близка к значению $1 \mathrm{~g} / \mathrm{cm}^{3}$. Различные значения
Параметры образцов

\begin{tabular}{c|c|c|c}
\hline $\begin{array}{c}\text { № } \\
\text { образца }\end{array}$ & $\begin{array}{c}\text { Содержание тех. } \\
\text { углерода, \% }\end{array}$ & $\rho, \Omega \cdot \mathrm{m}$ & $\Delta, \mathrm{m}$ \\
\hline 1 & 10 & $4 \cdot 10^{6}$ & 10.385 \\
2 & 15 & 25 & 0.026 \\
3 & 23 & 1.34 & $6.011 \cdot 10^{-3}$ \\
4 & 25 & 0.68 & $4.282 \cdot 10^{-3}$ \\
5 & 30 & 0.06 & $1.272 \cdot 10^{-3}$ \\
6 & 35 & 0.03 & $0.9 \cdot 10^{-3}$
\end{tabular}

удельного сопротивления $\rho$ достигали изменением содержания технического углерода в составе композита. Варьирование $\rho$ производилось в области перколяции. Удельное сопротивление измеряли четырехзондовым методом на образцах с размерами $2 \times 10 \times 120 \mathrm{~mm}$. Образцы используемые для регистрации спектров ЭПР имели форму, близкую к кубической. Их масса измерялась с помощью электронных весов, с точностью $3 \%$. По значениям массы и плотности определяли объем образцов. Спектры ЭПР регистрировали на спектрометpe PS 100.X при комнатной температуре на частоте $9.3 \mathrm{GHz}$.

Значения удельного объемного сопротивления $\rho$ образцов и глубина скин-слоя $\Delta$, рассчитанная по формуле (3), а также содержание технического углерода в массовых долях (\%), представлены в таблице.

\section{5. Результаты и их обсуждения}

Необходимо отметить, что значение g-фактора для всех образцов было равным 2.0. С увеличением содержания технического углерода в составе композита интенсивность линии поглощения возрастала. Изменение спектра ЭПР при увеличении $V$ для образца № 5

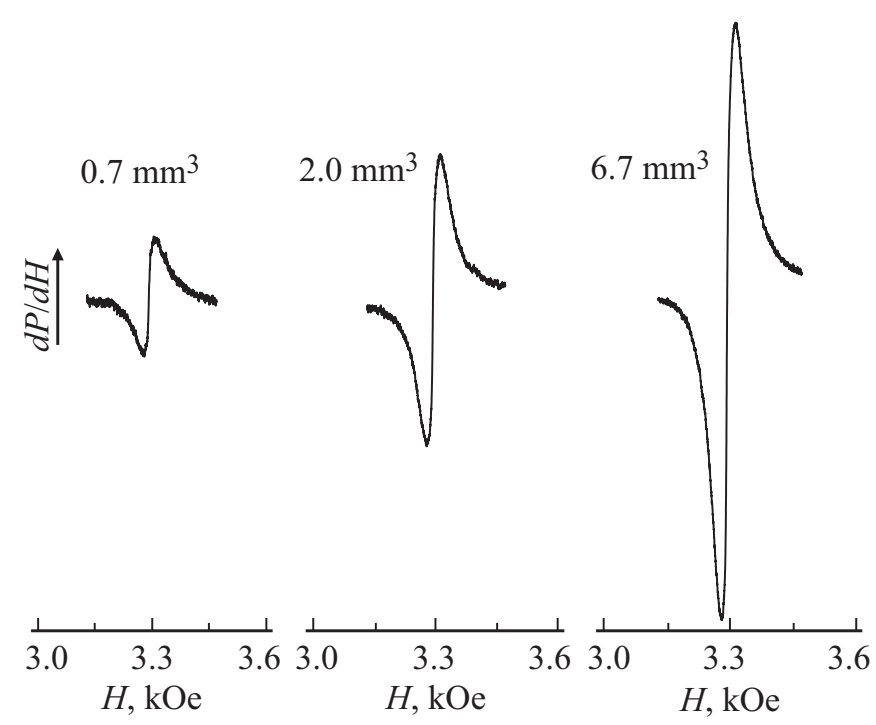

Рис. 1. Изменение спектра ЭПР при увеличении объема образца № 5. 
представлено на рис. 1. Видно, что интенсивность линии поглощения ЭПР с увеличением объема образца возрастает не пропорционально его значению.

Для определения влияния активных потерь в исследуемом образце на значение $\lambda$ использовался контрольный образец, спектр ЭПР которого был обусловлен ионами $\mathrm{Mn}^{2+}$ и имел сверхтонкую структуру. Вместе с контрольным, который был диэлектрическим и имел

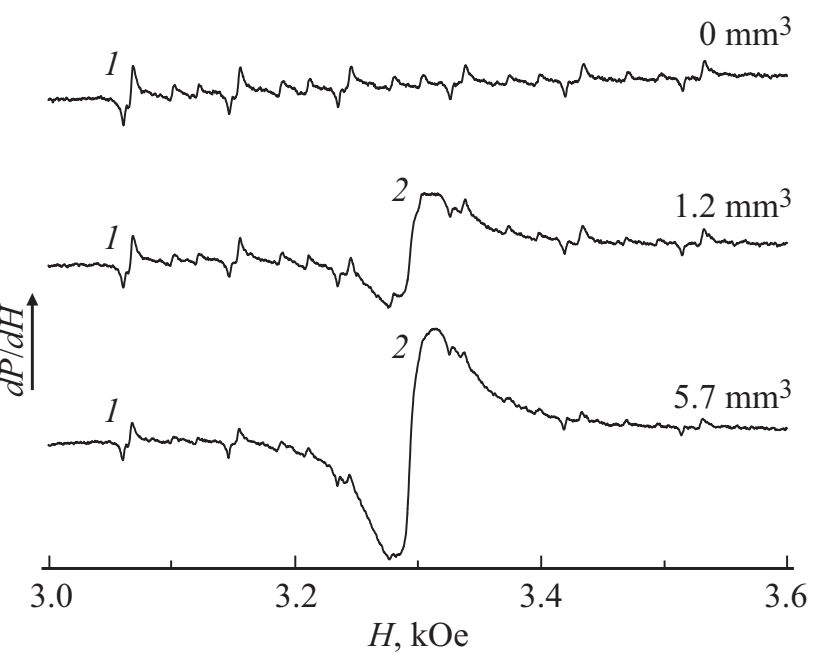

Рис. 2. Спектр линий поглощения контрольного (1) и исследуемого № 5 (2) образцов при различных объемах последнего.

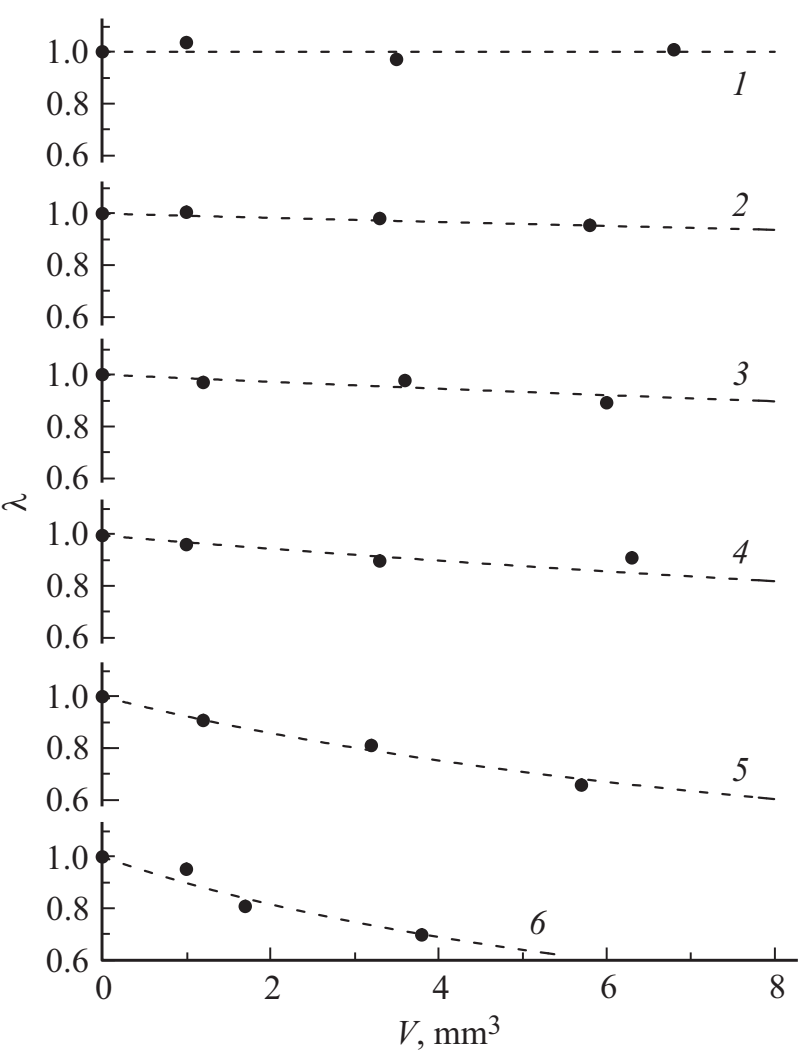

Рис. 3. Зависимости относительной интенсивности $I / I_{0}=\lambda$ контрольного образца от объема исследуемого. Точки эксперимент. Цифры у кривых - номера образцов.
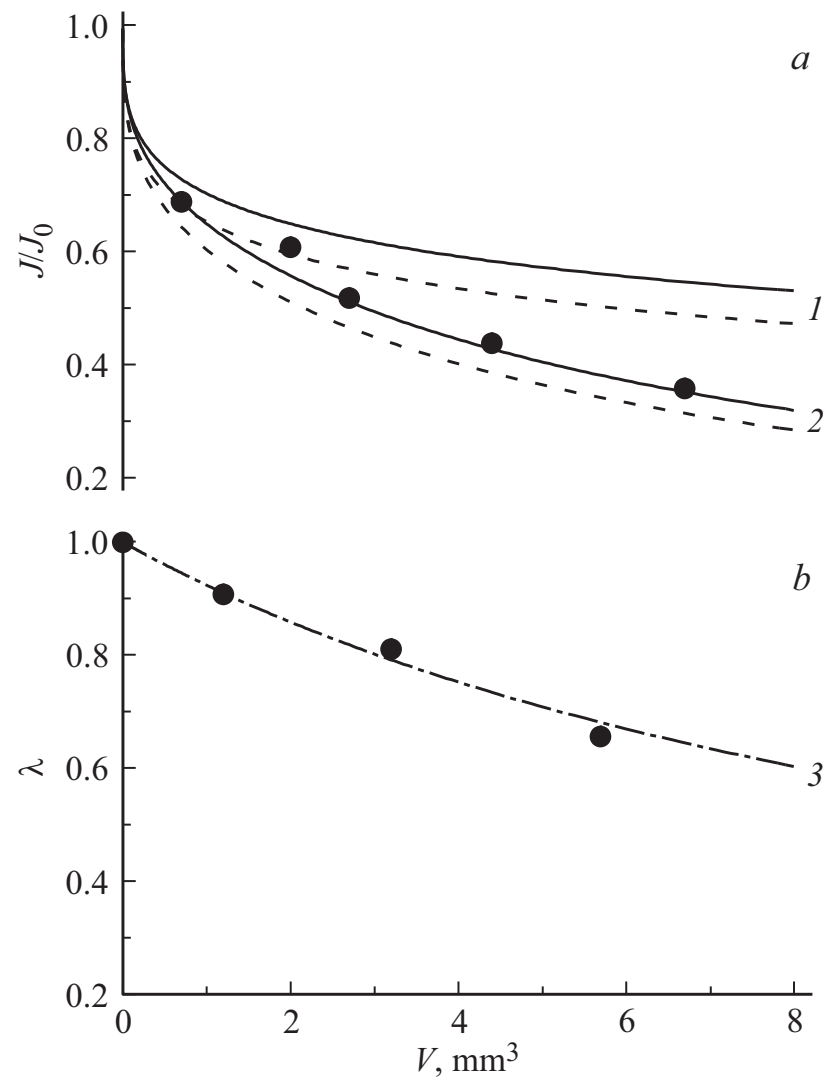

Рис. 4. $a-$ зависимость удельной интенсивности линии поглощения от объема для образца № 5: точки - эксперимент, кривые $(1)$ и (2) - расчет без учета и с учетом активных потерь соответственно. Сплошная кривая - образец кубической формы, штриховая - шарообразной. $b-$ экспериментальная зависимость $I / I_{0}=\lambda$ контрольного образца от $V$ исследуемого.

весьма малый объем, в резонатор поочередно помещались исследуемые образцы с разными значениями объема. Ряд линий сверхтонкой структуры спектра слева и справа от линии поглощения исследуемого образца хорошо разрешались (рис. 2). Это позволяло получить зависимости интенсивности линии поглощения ЭПР контрольного образца $I_{c}$ от объема исследуемых образцов с различными значениями удельного сопротивления. Значение $\lambda(V)$ принималось равным отношению $I_{c}$, когда в резонаторе вместе с контрольным находился исследуемый образец объемом $V$, к интенсивности $I_{c 0}$, в отсутствии последнего $\lambda(V)=I_{c} / I_{c 0}=I_{c}^{\prime} / I_{c 0}^{\prime}$. Из рис. 2 . видно, что увеличение объема исследуемого образца приводит к уменьшению амплитуды сигнала контрольного. Это обусловлено возрастанием влияния активных потерь в исследуемом образце на добротность $Q_{s}$, а следовательно и на $Q_{u}$.

На рис. 3 представлены экспериментальные зависимости коэффициента $\lambda$ от объема исследуемых образцов с разным значением удельного сопротивления. Штриховые линии являются аппроксимацией экспериментальных зависимостей $\lambda(V)$ зависимостью (10) при значении параметра $K$, обеспечивающем наилучшее согласование. 

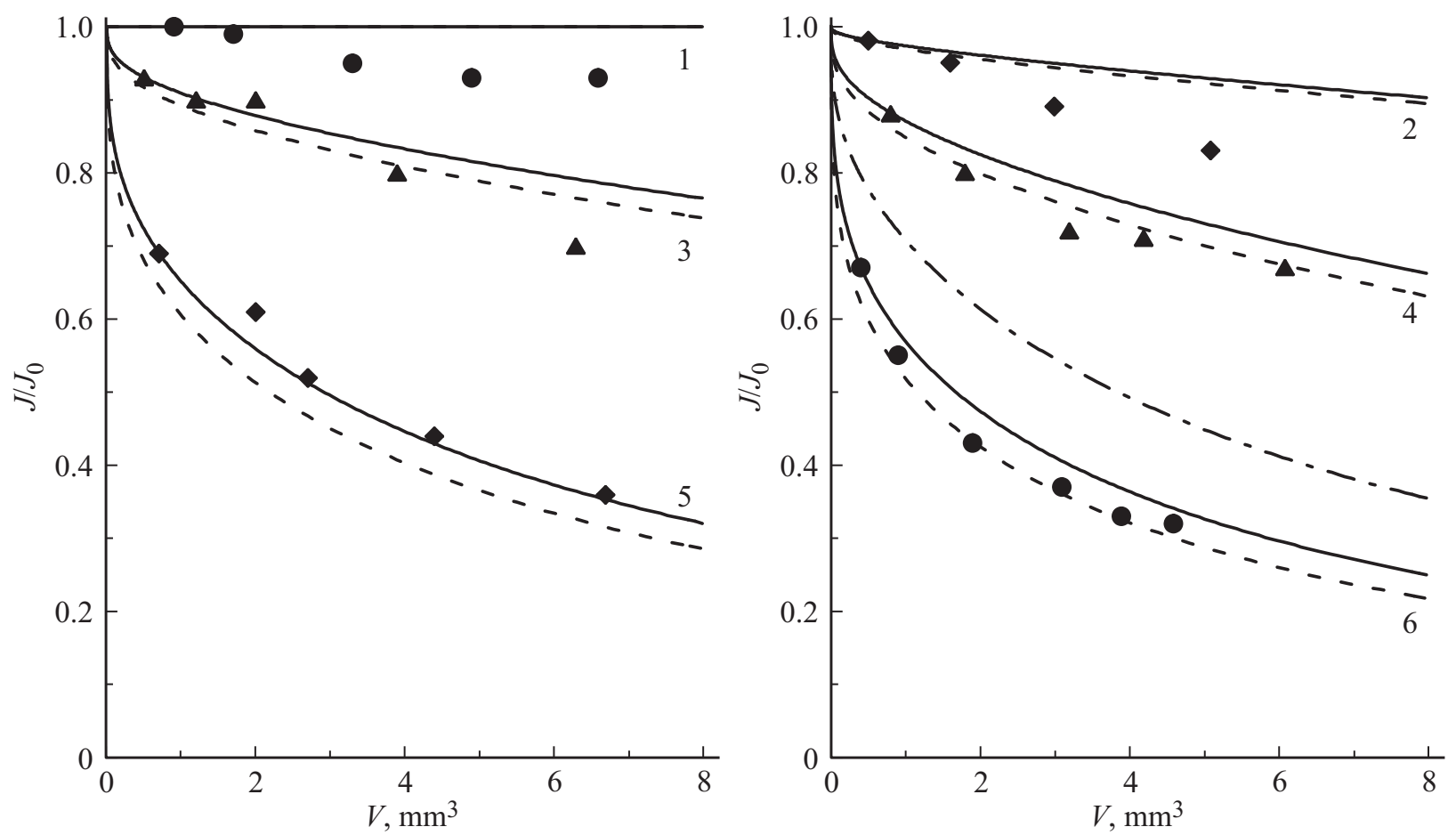

Рис. 5. Экспериментальные (точки) и расчетные зависимости удельной интенсивности от объема образца для различных значений $\rho$. Сплошная кривая - образец кубической формы, штриховая - шарообразной, штрих-пунктирная - расчет для образца № 6 без учета затухания сигнала при обратном прохождении. Цифры у кривых — номера образцов.

По этим значениям строились и расчетные зависимости $J / J_{0}=\beta(V) \cdot \lambda(V)$.

Из рис. 3 видно, что для образца № 1, имеющего большое значение $\rho$, величина $\lambda$ практически не зависит от $V$ и остается близкой к единице. С уменьшением $\rho$ влияние активных потерь на добротность системы резонатор-образец $Q_{u}$, а следовательно, и на интенсивность линии поглощения становится все более существенным. Заметим, что для образца № 6 с $\rho=0.03 \Omega \cdot \mathrm{m}$, при объеме $V$, большем чем $4 \mathrm{~mm}^{3}$ происходил срыв автоматической подстройки на резонансную частоту резонатора с образцом, вследствие чего спектр ЭПР зарегистрировать не удавалось.

На рис. 4, a для образца № 5 приведены экспериментальные зависимости удельной интенсивности от объема и расчетные $J / J_{0}$ учитывающие влияние лишь скин-эффекта (кривые 1) и расчетные, учитывающие также и влияние активных потерь (кривые 2). Видно, что учет одновременного влияния скин-эффекта и вклада в изменение добротности $Q_{u}$, обусловленного активными потерями в образце приводит к достаточно хорошему согласованию экспериментальных и расчетных результатов. На рис. 4, $b$ приведена экспериментальная зависимость от объема исследуемого образца, коэффициента $\lambda$, учитывающего влияние активных потерь.

На рис. 5. приведены экспериментальные $J / J_{0}$ (точки) и расчетные $J / J_{0}=\beta(V) \cdot \lambda(V)$ зависимости удельной интенсивности от объема образца для различных значений удельного объемного сопротивления. Расчетные зависимости получали, используя соответствующие значения $\lambda(V)$ (рис. 3 ) и $\beta(V)$, рассчитанные с помощью (6) или (7). Построение экспериментальных зависимостей производилось следующим образом. Значение удельной интенсивности для наименьшего измеренного объема образца ставилось в соответствие расчетному $J / J_{0}$ для такого же объема и, затем, в пересчете на данное значение наносились экспериментальные результаты относительной интенсивности для других значений $V$. Из рис. 5. следует, что если при больших значениях $\rho$ расчетные значения удельной интенсивности слабо зависят от объема образца, то с уменьшением $\rho$ влияние скин-эффекта и изменения добротности системы $Q_{u}$, обусловленное влиянием активных потерь на интенсивность линии поглощения становится весьма существенным. Видно, что экспериментальные и расчетные результаты достаточно хорошо согласуются между собой.

Нами также был проведен расчет $\beta(V)$, не учитывающий затухание сигнала поглощения при обратном прохождении от элемента объема $d V$ на глубине $x$ к поверхности образца. Зависимость удельной интенсивности $J / J_{0}=\beta(V) \cdot \lambda(V)$ для образца № 6 кубической формы, когда значения $\beta(V)$ рассчитывались как

$$
\beta(V)=\frac{24}{a^{3}} \int_{0}^{a / 2} x^{2} \exp \left[\frac{-2\left(\frac{a}{2}-x\right)}{\Delta}\right] d x,
$$

представлена на рис. 5 (штрих-пунктирная кривая). Видно, что расчет $\beta(V)$ согласно выражению (7), то 
есть с учетом затухания сигнала поглощения при обратном прохождении, гораздо лучше согласуется с экспериментом.

Некоторое несоответствие расчетных и экспериментальных результатов, наиболее заметно проявляющееся в образцах с малым значением $\rho$ может быть обусловлено дополнительным влиянием на поглощение и отражение микроволнового поля агломератов технического углерода в структуре композита. Такие агломераты обладают заметной электропроводностью в их внутренней области, но не участвуют в образовании цепочек проводимости, а следовательно не влияют на значение $\rho$. Они начинают оказывать влияние на удельную интенсивность уже при концентрациях 5-10\% технического углерода, то есть достаточно далеких от области перколяции и при которых удельное сопротивление велико.

\section{6. Выводы}

Таким образом, на основе результатов полученных в настоящей работе можно сделать следующие выводы.

1. Предложен подход, позволяющий учитывать влияние скин-эффекта на интенсивность линии ЭПР в полупроводящих веществах. Показано, что в веществах с заметной проводимостью удельная интенсивность линии поглощения может в разы уменьшатся в результате действия скин-эффекта.

2. Большое влияние на интенсивность линий поглощения ЭПР оказывают активные потери в образце, величина которых существенно зависит от его объема.

3. Получено удовлетворительное соответствие расчетных и экспериментальных результатов по зависимости удельной интенсивности линий поглощения ЭПР от объема образца в широком интервале значений удельного объемного сопротивления в углеродсодержащих полимерных композитах.

\section{Финансирование работы}

Работа выполнена при финансовой поддержке Российского фонда фундаментальных исследований и Правительства Республики Мордовия в рамках научного проекта № 18-48-130015 p_a.

\section{Конфликт интересов}

Авторы заявляют, что у них нет конфликта интересов.

\section{Список литературы}

[1] J. Aneli, G. Zaikov, O. Mukbaniani. Chem. Chem. Tech. 5, 1, 75 (2011).

[2] M. Rahaman, T.K. Chaki, D. Khastgir. J. Mater. Sci. 48, 7466 (2013).

[3] В.А. Марков, Л.Б. Кандырин, А.В. Марков. Вестник МИТХТ 8, 6, 103 (2013).

[4] Т.А. Борукаев, Д.С. Гаев. Прикл. физика 5, 76 (2017).
[5] W. Zhang, A.A. Dehghani-Sanij, R.S. Blackburn. J. Mater. Sci. 42, 3408 (2007).

[6] В.Е. Живулин, Л.А. Песин, Д.В. Иванов. ФТТ 58, 87 (2016).

[7] M. Chipara, W.J. Brittain, K.T. Lau, M.D. Chipara. Adv. Mater. Res. 45-50, 1165 (2008).

[8] A.A. Monge, N. Ferrer-Anglada, V. Lloveras, J. VidalGancedo, S. Roth. Phys. Status Solidi B 248, 2564 (2011).

[9] M. Chipara, J.M. Zaleski, D. Hui, C. Du, N. Pan. J. Polym. Sci. Pol. Phys. 43, 3406 (2005).

[10] Дж. Вертц, Дж. Болтон. Теория и практические приложения метода ЭПР. Мир, М. (1975). 552 с.

[11] А.М. Зюзин, В.Н. Ваньков, В.В. Радайкин. Письма в ЖТФ 17, 23, 65 (1991).

[12] А.М. Зюзин, С.Н. Сабаев, А.В. Куляпин. ФТТ 45, 2208 (2003).

[13] Ж. Винтер. Магнитный резонанс в металлах. Мир, М. (1976). 288 c.

[14] F.J. Dyson. Phys. Rev. 98, 349 (1955).

[15] Ч. Пул. Техника ЭПР-спектроскопии. Мир, М. (1970). $435 \mathrm{c}$.

[16] С.А. Альтшулер, Б.М. Козырев. Электронный парамагнитный резонанс соединений элементов промежуточных групп. Наука, М. (1972). 670 с.

Редактор Т.Н. Василевская 